\title{
Increased BTLA and HVEM in gastric cancer are associated with progression and poor prognosis
}

\author{
This article was published in the following Dove Press journal: \\ OncoTargets and Therapy \\ 16 February 2017 \\ Number of times this article has been viewed
}

\section{Xiuwen $\operatorname{Lan}^{1, *}$ \\ Sen $\mathrm{Li}^{1}$,* \\ Hongyu Gao' \\ Abiyasi Nanding ${ }^{2}$ \\ Lina Quan ${ }^{3}$ \\ Chunyan Yang ${ }^{4}$ \\ Shaohua Ding ${ }^{2}$ \\ Yingwei Xue'}

'Department of Gastroenterological Surgery, ${ }^{2}$ Department of Pathology,

${ }^{3}$ Department of Hematology,

The Affiliated Tumor Hospital,

${ }^{4}$ Department of Epidemiology and

Biostatistic, Public Health School,

Harbin Medical University, Harbin,

People's Republic of China

*These authors contributed equally to this work
Correspondence: Yingwei Xue Department of Gastroenterological Surgery, The Affiliated Tumor Hospital, Harbin Medical University, 150 Haping Road, Nangang District, Harbin I5008I, People's Republic of China Tel/fax +86 45I 86298303

Email xyw80I@I63.com
Purpose: Deregulation of immune checkpoint molecules by tumor cells is related to immune escape. This study was conducted to investigate the relationship between the appearance of B- and T-lymphocyte attenuator (BTLA) and its ligand herpesvirus entry mediator (HVEM) with the prognosis in gastric cancer patients.

Patients and methods: A total of 136 patients with curative gastrectomy were included. The expression of BTLA and HVEM was detected by immunohistochemistry, and its correlation with the clinical significance of gastric cancer was further analyzed.

Results: The positivity of BTLA and HVEM was detected in 74.3\% (101/136) and 89.0\% $(121 / 136)$ of the gastric cancer specimens, respectively. A high expression of BTLA and HVEM was detected, respectively, in $28.7 \%(39 / 136)$ and $44.9 \%(61 / 136)$ of the specimens. Characteristics analysis showed that the high expression of BTLA was significantly associated with lymph node metastasis $(P=0.030)$. Similarly, the high expression of HVEM was also significantly correlated with lymph node metastasis $(P=0.007)$ and depth of invasion $(P=0.011)$. In addition, there was a positive correlation between the expression of BTLA and HVEM in gastric cancer specimens $(r=0.245, P=0.004)$. Univariate analysis revealed that the high expression of BTLA and HVEM was associated with overall survival of patients along with tumor size, Borrmann type, depth of invasion, lymph node metastasis, and histological grade $(P<0.05)$. Multivariate analysis established that the high expression of HVEM $(P=0.010)$, depth of invasion $(P=0.001)$, lymph node metastasis $(P<0.001)$, and histological grade $(P=0.027)$ were independent prognostic factors associated with overall survival in patients with gastric cancer.

Conclusion: The increased BTLA and HVEM levels correlate with the development and poor prognosis of gastric cancer. HVEM is an important prognostic indicator, and BTLA/HVEM pathway is considered to be a promising candidate for immunotherapy of gastric cancer.

Keywords: gastric cancer, B- and T-lymphocyte attenuator, herpesvirus entry mediator, prognostic

\section{Introduction}

Gastric cancer is one of the most fatal malignancies and the third leading cause of mortality. ${ }^{1}$ The immune system has an important impact on the development of gastric cancer. Immune checkpoints, existing in the molecules of the immune system, function as a regulator of immune signals and play an important role in T-cell activation as well as in the recognition and destruction of tumor cells. However, tumor cells can evade immune surveillance and promote their own survival through a distinct mechanism, including expression of ligands for co-inhibitory receptors. ${ }^{2}$ Checkpoint inhibitors, especially CTLA-4 and PD-1 blockades, as the new direction of cancer immunotherapy, have achieved a breakthrough in previous studies. ${ }^{3,4}$ Therefore, further 
experimental and clinical research on other candidate checkpoint inhibitors would be of great significance for exploiting potent immunotherapy.

BTLA is a novel checkpoint co-inhibitory receptor belonging to the CD28 superfamily (also known as the Immunoglobulin [Ig] superfamily), which appears on a wide range of immune cells, including $\mathrm{T}, \mathrm{B}, \mathrm{NK}$, and other cells. ${ }^{5}$ BTLA contains an immunoreceptor tyrosine-based inhibitory motif (ITIM) and an immunoreceptor tyrosine-based switch motif(ITSM), structurally similar to PD- 1 and CTLA-4. ${ }^{6}$ Its ligand HVEM (also known as TNFRSF14) is a member of the tumor necrosis factor receptor (TNFR) superfamily. HVEM was found to be widely present on hematopoietic cells and a variety of parenchymal cells, such as breast, melanoma, esophageal, colorectal, and ovarian cancer cells. ${ }^{2,7-10}$ In the past decade, HVEM has emerged as a bidirectional immune molecule that is involved in the inhibition or stimulation of T cells by binding to BTLA or LIGHT (TNFSF14). ${ }^{11}$ The combination of BTLA to cysteine-rich domains 1 (CRD1) of HVEM makes this pathway an important cross talk between Ig and TNF superfamily. ${ }^{12,13}$ Furthermore, the BTLA/HVEM pathway appears to be a new possible approach of immune escape and is considered to be a critical factor in the physiological process of inflammation and tumorigenesis. ${ }^{14,15}$

So far, little attention has been paid to the expression of BTLA in gastric cancer, and the expression of HVEM in human gastric cancer tissues has not been addressed. This study aimed to determine the expression of BTLA and HVEM in human gastric cancer specimens by using immunohistochemistry (IHC) detection, and to investigate its relationship with clinicopathological characteristics and prognostic factors.

\section{Materials and methods}

\section{Patients and follow-up}

Included in the present study were a total of 136 patients, who were histologically confirmed with gastric adenocarcinoma and underwent gastrectomy, from June 2009 to May 2010, at the Department of Gastroenterological Surgery of the Affiliated Tumor Hospital of Harbin Medical University (Harbin, People's Republic of China). The gastric adenocarcinoma tissues, along with clinicopathological data and follow-up information, were obtained from those patients. In addition, 20 matched adjacent normal tissues (at least $5 \mathrm{~cm}$ from the tumor site) were also collected from those patients during surgery. Among these 136 patients, 95 (69.9\%) were men and $41(30.1 \%)$ were women. The median age was 58 years (ranging from 23 to 77 years). None of these patients received neoadjuvant chemotherapy, chemoradiotherapy, or immunotherapy. Patients with distant metastases, remnant gastric cancers, or other gastric malignancies such as lymphomas and gastrointestinal stromal tumors were excluded from this analysis. The stage of gastric cancer was determined according to the TNM staging system of the 7th UICC/AJCC manual. ${ }^{16}$ The survival duration was calculated from the time of surgery to death or to the last follow-up date (January 31 , 2016). The median follow-up time was 36 months, ranging from 6 to 76 months. The present study was approved by the Clinical Research Ethics Committee of The Affiliated Tumor Hospital of Harbin Medical University (Harbin, People's Republic of China). All patients gave their written informed consent for participation in this study.

\section{Immunohistochemistry}

In accordance with the manufacturer's instructions, IHC staining for BTLA and HVEM was performed by the application of the Two-Step IHC Detection Reagent (PV-6001) Kit (ZhongShan Golden Bridge Biological Technology Inc, Beijing, People's Republic of China). In brief, tissues were formalin fixed, processed, and embedded in paraffin wax. Then $4-\mu \mathrm{m}$-thick sections were baked, deparaffinized in xylene, and rehydrated. Slides were then incubated with 3\% $\mathrm{H}_{2} \mathrm{O}_{2}$ for 10 min to block endogenous peroxidase activity. After being autoclaved for $2 \mathrm{~min}$ in citrate buffer ( $\mathrm{pH}$ 6.0) for antigen retrieval, each section was incubated with rabbit polyclonal antibody against BTLA (1:400 dilution, ab181406, Abcam, Cambridge, MA, USA) or rabbit polyclonal antibody against HVEM (1:800 dilution, ab47677, Abcam) at $4{ }^{\circ} \mathrm{C}$ overnight. After being incubated with secondary antibodies at room temperature for $20 \mathrm{~min}$, each section was detected with diaminobenzidine (DAB) reagent sets for 2-3 min, and then counterstained with hematoxylin. Finally, the sections were dehydrated by gradient alcohol and covered with a coverslip.

Protein expression in a specimen was recorded as "Negative" if the percentage of positively stained cancer cells showed $<5 \%$. Total immunostaining was scored by combining the percentage and intensity of positively stained tumor cells. ${ }^{17}$ The percentage of positively stained tumor cells was classified as follows: $0,<5 \% ; 1,5 \%-25 \% ; 2,26 \%-50 \%$; and $3,>50 \%$. The staining intensity was graded as follows: 0 , negative; 1 , weak staining; 2 , moderate staining; and 3 , strong staining. The final score was calculated by multiplying the positive proportion score and the staining intensity score (the total score ranging from 0 to 9 ). The specimen with a final 
score of $\geq 4$ was classified as high expression, but otherwise as low expression. The staining on each slide was evaluated by two independent pathologists in a blinded manner, and a final consensus was reached.

\section{Statistical analysis}

The analysis was performed using SPSS 19.0 for windows (version 19.0, IBM Corp., Armonk, NY, USA). The relationship between BTLA and HVEM expression and clinicopathological factors was tested using the chi-square test. Correlation between expression levels was studied using the Spearman's rank correlation analysis. Survival curves were calculated using the Kaplan-Meier method. A log-rank test was applied for univariate analysis, and a stepwise Cox proportional hazards model was utilized for multivariate analysis to identify independent prognostic factors. $P<0.05$ was considered to be statistically significant.

\section{Results \\ Expression status of BTLA and HVEM in gastric tissues}

The expression of BTLA and HVEM proteins in gastric cancer samples and adjacent normal tissues was analyzed by IHC. The positive BTLA and HVEM staining was predominantly localized within the cytoplasmic of gastric cancer cells. On the contrary, there was no staining observed in the adjacent normal tissues (0/20) (Figure 1). Positive BTLA and HVEM expression was detected in $101(74.3 \%)$ and
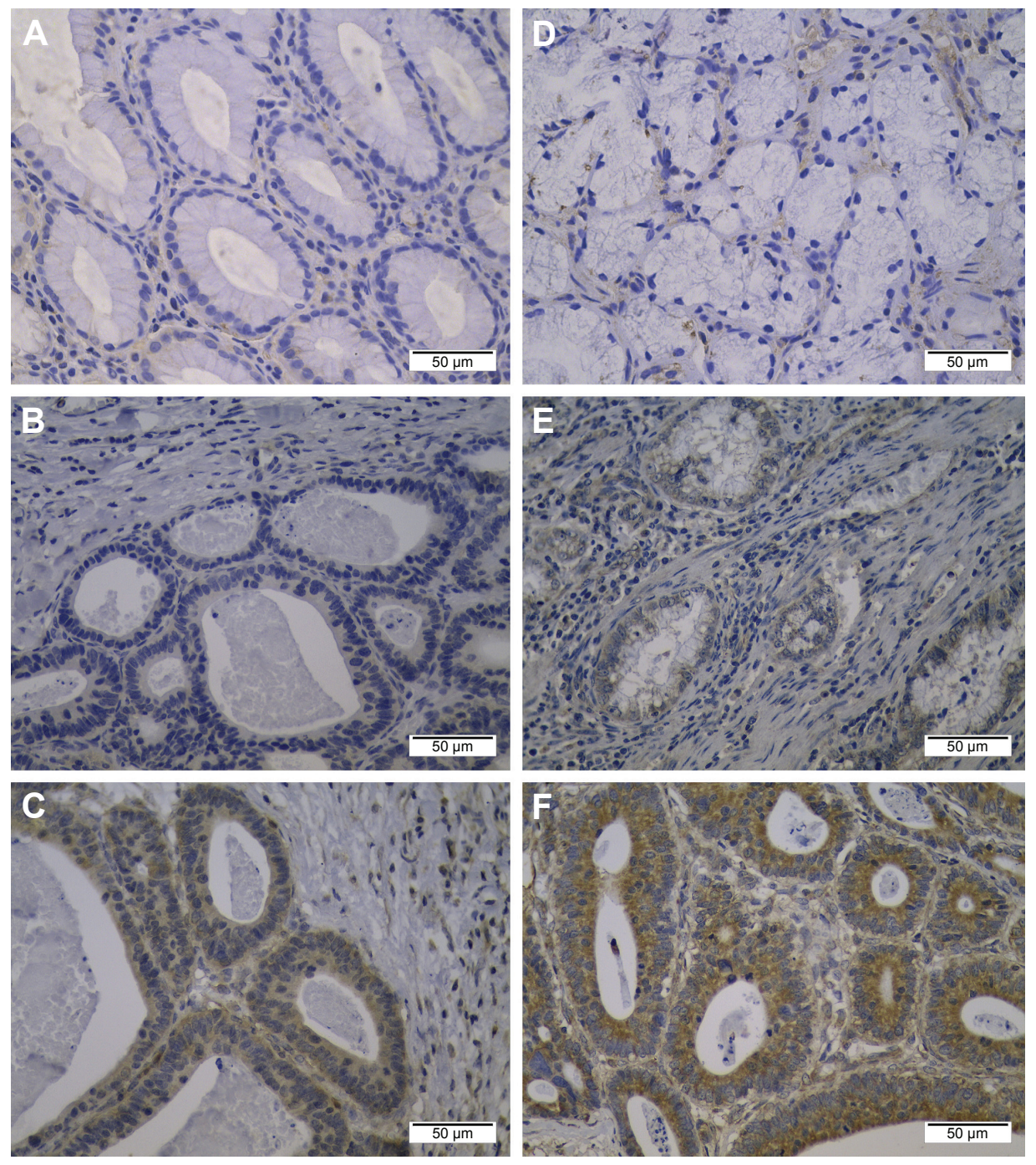

Figure I Representative picture of immunohistochemical staining of BTLA and HVEM in gastric tissues.

Notes: (A) Negative BTLA expression in adjacent normal specimen. (B) Low BTLA expression in gastric cancer. (C) High BTLA expression in gastric cancer. (D) Negative HVEM expression in adjacent normal specimen. (E) Low HVEM expression in gastric cancer. (F) High HVEM expression in gastric cancer. Magnifications: $\times 400$.

Abbreviations: BTLA, B- and T-lymphocyte attenuator; HVEM, herpesvirus entry mediator. 
$121(89.0 \%)$ of the 136 specimens, respectively. However, the high BTLA and HVEM expression was detected in 39 $(28.7 \%)$ and 61 (44.9\%), respectively.

\section{Association between BTLA and HVEM expression and clinicopathological variables in gastric cancer}

Among the 136 gastric cancer specimens examined, the high expression of BTLA exhibited a significant correlation with the lymph node metastasis $(P=0.030)$. Likewise, the high expression of HVEM was significantly correlated with the lymph node metastasis $(P=0.007)$ and depth of invasion $(P=0.011)$. In addition, the increased HVEM expression was likely to be correlated with the Borrmann type although there was no statistically significant difference $(P=0.053)$. However, HVEM expression was found to have no statistically significant difference among the factors of age, gender, tumor size, tumor location, or histological grade $(P>0.05)$. Similarly, BTLA expression was also not associated with any of the other clinical parameters (Table 1). Spearman's correlation analysis showed that the high expression of BTLA was positively correlated with the high level of HVEM $(r=0.245, P=0.004)$ (Table 2).
Clinical impact of BTLA and HVEM on survival of patients with gastric cancer

Kaplan-Meier survival curves demonstrated that the high expressions of BTLA as well as HVEM were markers of poor prognosis in gastric cancer patients $(P=0.001$ and $P<0.001)$ (Figures 2 and 3). The 5-year overall survival (OS) rate of patients with high and low BTLA expressions were 17.9\% and $48.3 \%$, respectively. Similarly, in patients with increased HVEM expression, the 5-year OS rate was $19.1 \%$, which was poorer than that of its counterpart (55.9\%). There was no statistically significant difference in the 5-year OS rate for patients between BTLA and HVEM $(P=0.300)$.

As shown in Table 3, the univariate analysis identified seven variables as having a statistically significant association with the OS of all patients, which included a high expression of both BTLA and HVEM ( $P=0.001$ and $P<0.001$, respectively), tumor size $(P=0.031)$, Borrmann type $(P=0.018)$, depth of invasion $(P<0.001)$, lymph node metastasis $(P<0.001)$, and histological grade $(P<0.013)$. In addition, a multivariate Cox proportional hazard model was used to assess those seven variables. The results of the assessment showed that independent prognostic factors for OS included

Table I Association between BTLA and HVEM expression and clinicopathological factors of gastric cancer patients

\begin{tabular}{|c|c|c|c|c|c|c|c|}
\hline \multirow[t]{2}{*}{ Variable } & \multirow[t]{2}{*}{ Case $\mathbf{N}$} & \multicolumn{3}{|l|}{ BTLA } & \multicolumn{3}{|l|}{ HVEM } \\
\hline & & Low N (\%) & High N (\%) & $P$-values ${ }^{a}$ & Low N (\%) & High N (\%) & $P$-values ${ }^{\mathrm{a}}$ \\
\hline Age, years & & & & 0.842 & & & 0.269 \\
\hline$<60$ & 74 & $54(73.0)$ & $20(27.0)$ & & $44(59.5)$ & $30(40.5)$ & \\
\hline$\geq 61$ & 62 & $43(69.4)$ & $19(30.6)$ & & $31(50.0)$ & $31(50.0)$ & \\
\hline Gender & & & & 0.642 & & & 0.545 \\
\hline Male & 95 & 69 (72.6) & $26(27.4)$ & & $54(56.8)$ & $4 \mathrm{I}(43.2)$ & \\
\hline Female & 41 & $28(68.3)$ & $13(31.7)$ & & $21(5 \mid .2)$ & $20(48.8)$ & \\
\hline Tumor size, cm & & & & 0.244 & & & 0.141 \\
\hline$<5$ & 63 & $48(76.2)$ & $15(23.8)$ & & $39(61.9)$ & $24(38.1)$ & \\
\hline$\geq 5$ & 73 & $49(67.1)$ & $24(32.9)$ & & $36(49.3)$ & 37 (50.7) & \\
\hline Location & & & & 0.458 & & & 0.163 \\
\hline Upper third & 16 & I3 (8I.2) & $3(18.8)$ & & $8(50.0)$ & $8(50.0)$ & \\
\hline Middle third & 37 & $24(64.9)$ & $13(35.1)$ & & $16(43.2)$ & $21(56.8)$ & \\
\hline Lower third & 83 & $60(72.3)$ & $23(27.7)$ & & $51(61.4)$ & $32(38.6)$ & \\
\hline Borrmann type & & & & 0.221 & & & 0.053 \\
\hline $\mathrm{I} / \mathrm{II}$ & 38 & 30 (78.9) & $8(2 \mid . I)$ & & $26(68.4)$ & $12(31.6)$ & \\
\hline III/IV & 98 & $67(68.4)$ & $31(31.6)$ & & $49(50.0)$ & $49(50.0)$ & \\
\hline Depth of invasion & & & & 0.284 & & & 0.011 \\
\hline $\mathrm{TI}-2$ & 29 & $23(79.3)$ & $6(20.7)$ & & $22(75.9)$ & $7(24.1)$ & \\
\hline T3-4 & 107 & $74(69.2)$ & $33(30.8)$ & & $53(49.5)$ & $54(50.5)$ & \\
\hline Lymph node metastasis & & & & 0.030 & & & 0.007 \\
\hline No & 43 & $36(83.7)$ & $7(16.3)$ & & $31(72.1)$ & $12(27.9)$ & \\
\hline Yes & 93 & $61(65.6)$ & $32(34.4)$ & & $44(47.3)$ & $49(52.7)$ & \\
\hline Histological grade & & & & 0.556 & & & 0.695 \\
\hline Well/moderate & 47 & $35(74.5)$ & $12(25.5)$ & & $27(57.4)$ & $20(42.6)$ & \\
\hline Poor & 89 & $62(69.7)$ & $27(30.3)$ & & $48(53.9)$ & $4 I(46.1)$ & \\
\hline
\end{tabular}

Note: ${ }^{a} \chi^{2}$ test.

Abbreviations: BTLA, B- and T-lymphocyte attenuator; HVEM, herpesvirus entry mediator. 
Table 2 Relationship between BTLA and HVEM expression in gastric cancer

\begin{tabular}{llllll}
\hline BTLA & HVEM & & & r-value & P-value $^{\mathbf{a}}$ \\
\cline { 2 - 4 } & High & Low & Total & & \\
\hline High & 25 & 14 & 39 & 0.245 & 0.004 \\
Low & 36 & 61 & 97 & & \\
Total & 61 & 75 & & & \\
\hline
\end{tabular}

Note: aSpearman's rank.

Abbreviations: BTLA, B- and T-lymphocyte attenuator; HVEM, herpesvirus entry mediator.

the high expression of HVEM $(P=0.010$, hazard ratio $[\mathrm{HR}]=1.857,95 \%$ confidence interval $[\mathrm{CI}]: 1.161-2.970)$ accompanied by depth of invasion $(P=0.001, \mathrm{HR}=2.281$, 95\% CI: 1.418-3.671), lymph node metastasis $(P<0.001$, HR $=1.499,95 \%$ CI: $1.200-1.874)$, and histological grade $(P=0.027, \mathrm{HR}=1.334,95 \%$ CI: $1.033-1.723)$ (Table 3$)$.

\section{Discussion}

Worldwide, gastric cancer is still one of the most pathogenic and lethal malignancies. The development of gastric cancer is associated with various factors such as environmental factors, infection, genetic factors, and immune response. The immune system can restrain or destroy the development of cancer cells. However, cancer cells can escape immune surveillance by exploiting the immune checkpoint pathway. The importance of checkpoint pathway mediated immune escape in cancer development has been highlighted by encouraging the clinical efficacy of hematologic malignancies and solid tumors. ${ }^{18,19}$ In recent years, increasing attention has been focused on the immune checkpoints and their correlation with gastric cancer. ${ }^{20}$ Accordingly, the combination of detection

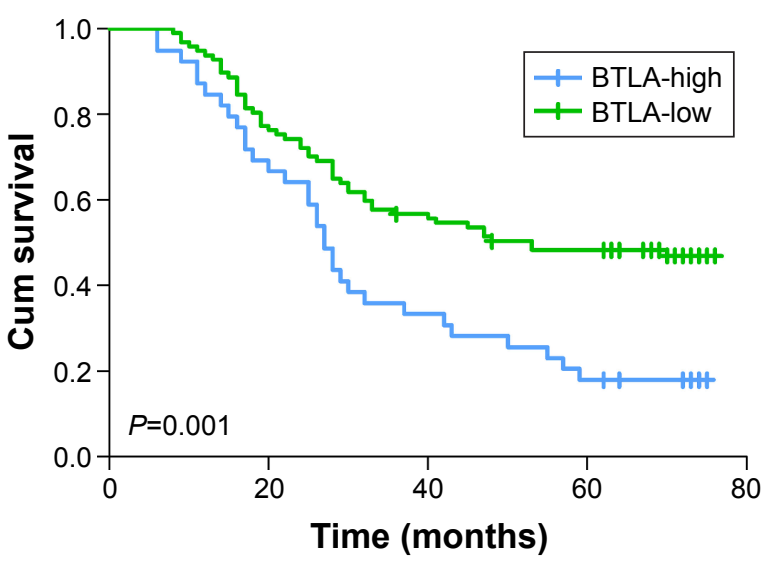

Figure 2 Kaplan-Meier curves of OS based on BTLA expression in patients with gastric cancer.

Notes: Higher expression of BTLA was closely correlated with poor OS $(P=0.00 \mathrm{I})$.

Abbreviations: OS, overall survival; BTLA, B- and T-lymphocyte attenuator; Cum, cumulative.

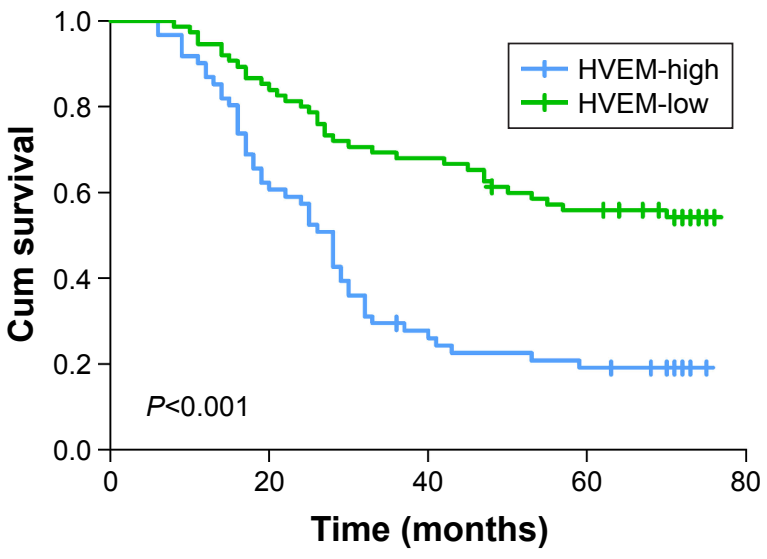

Figure 3 Kaplan-Meier curves of OS based on HVEM expression in patients with gastric cancer.

Notes: Higher expression of HVEM was closely correlated with poor OS $(P<0.00$ I).

Abbreviations: OS, overall survial; HVEM, herpesvirus entry mediator; Cum, cumulative.

of candidate checkpoints and their ligands may supply a new way to utilize immunotherapy in gastric cancer. Therefore, the present experiment was devised to detect the expression of BTLA and HVEM, which may contribute to the treatment of gastric cancer.

The expression of HVEM on gastric cancer and adjacent normal tissues was first detected using IHC, and HVEM along with checkpoint BTLA was upregulated in most gastric cancers compared with normal controls. This upregulation was associated with worse clinical response, which indicated advanced lymph node metastasis and depth of invasion than that of its counterpart. In addition, increased expression of HVEM was likely to be associated with worse Borrmann types. The results are generally consistent with the high expression of HVEM in esophageal cancer, which was involved in tumor invasion and lymph node metastasis. ${ }^{9}$ A previous study reported that soluble HVEM in the sera of gastric cancer patients was significantly higher than that of normal control, which was argued to be produced by ectodomain shedding rather than by simple secretion. ${ }^{21}$ Another study found that HVEM was overexpressed in colorectal cancer tissues, being correlated with tumor invasion and pathological stage, but being inversely correlated with the presence of tumor-infiltrating T cells. ${ }^{7}$ On the basis of recent reports and the present study, it is suggested that HVEM expression might be associated with progression and growth pattern of tumors. What is more, BTLA expression was associated with lymph node metastasis. In addition, the high expression of HVEM observed in gastric cancer was significantly correlated with BTLA expression since BTLA was initially recognized by a wide range of immune cells, 
Table 3 Univariate and multivariate analysis of the prognostic factors in 136 patients with gastric cancer

\begin{tabular}{|c|c|c|c|c|}
\hline \multirow[t]{2}{*}{ Variable } & \multicolumn{2}{|l|}{ Univariate } & \multicolumn{2}{|l|}{ Multivariate } \\
\hline & Mean survival (months) $(95 \% \mathrm{Cl})$ & $P$-values ${ }^{a}$ & HR (95\% Cl) & $P$-values \\
\hline Age, years & & 0.694 & & \\
\hline$<60$ & $45.770(39.86 I-5 I .679)$ & & & \\
\hline$\geq 61$ & $44.5 \mid 2(37.794-51.230)$ & & & \\
\hline Gender & & 0.559 & & \\
\hline Male & $46.116(40.664-51.568)$ & & & \\
\hline Female & $42.755(35.346-50.164)$ & & & \\
\hline Tumor size, $\mathrm{cm}$ & & 0.031 & & \\
\hline$<5$ & 49.748 (43.019-56.477) & & & \\
\hline$\geq 5$ & $41.216(35.496-46.935)$ & & & \\
\hline Location & & 0.256 & & \\
\hline Upper third & $50.035(36.890-63.179)$ & & & \\
\hline Middle third & $39.54 I(31.480-47.60 I)$ & & & \\
\hline Lower third & $46.682(40.915-49.869)$ & & & \\
\hline Borrmann type & & 0.018 & & \\
\hline $\mathrm{I} / \mathrm{II}$ & $52.632(44.09|-6| . \mid 72)$ & & & \\
\hline III/IV & $42.359(37.265-47.453)$ & & & \\
\hline Depth of invasion & & $<0.001$ & & 0.001 \\
\hline TI-2 & $67.932(61.230-74.635)$ & & I ref & \\
\hline T3-4 & $39.00 \mathrm{I}(34.30 \mathrm{I}-43.700)$ & & $2.28 I(|.4| 8-3.67 \mid)$ & \\
\hline Lymph node metastasis & & $<0.001$ & & $<0.001$ \\
\hline No & $66.450(61.032-71.868)$ & & I ref & \\
\hline Yes & $35.442(30.598-40.286)$ & & $1.499(1.200-1.874)$ & \\
\hline Histological grade & & 0.013 & & 0.027 \\
\hline Well/moderate & $53.160(45.886-60.434)$ & & I ref & \\
\hline Poor & 41.031 (35.634-46.428) & & $1.334(1.033-1.723)$ & \\
\hline BTLA expression & & 0.001 & & \\
\hline Low & $49.537(44.191-54.883)$ & & & \\
\hline High & $34.987(27.835-42.139)$ & & & \\
\hline HVEM expression & & $<0.001$ & & 0.010 \\
\hline Low & $54.972(49.226-60.718)$ & & I ref & \\
\hline High & $33.354(27.630-39.077)$ & & $1.857(1.16 \mathrm{I}-2.970)$ & \\
\hline
\end{tabular}

Notes: aLog-rank test; ${ }^{b}$ Cox regression test.

Abbreviations: BTLA, B- and T-lymphocyte attenuator; $\mathrm{Cl}$, confidence interval; HR, hazard ratio; HVEM, herpesvirus entry mediator; ref, reference.

which, accordingly, determined the biological behaviors of cancer cells. In fact, it could also be expressed by cancer cells, which was evidenced by ectopic expression of BTLA on gastric cancer. ${ }^{17}$ Previous study ${ }^{2}$ and $\mathrm{M}^{\prime} \mathrm{Hidi}$ et al ${ }^{22}$ also demonstrated that the presentation of HVEM/BTLA cis complex on the same B-chronic lymphocytic leukemia (B-CLL) cell could prevent the trans interactions with the other ligands of HVEM expressed on T cells, and the coexpression of BTLA and HVEM on CLL cells triggers an ineffective autocrine inhibitory loop.

In the present study, survival analysis demonstrated that the elevation of the two immune molecules exerted a negative effect on patient survival. Notably, multivariate analysis indicated that increased HVEM expression was an independent predictor for poorer prognosis. A previous study on melanoma demonstrated that the expression of HVEM was correlated with disease outcomes. ${ }^{14}$ Launay et a ${ }^{23}$ identified a higher frequency of TNFRSF14 gene mutations present in follicular lymphoma, which might affect the binding affinity to BTLA and then might lead to better clinical outcomes. Immune regulation is considered an important hallmark of tumor initiation and progression and consequently affects patient outcomes. Previously, studies have also associated the checkpoint BTLA/HVEM pathway with the regulation of tumor immune response. For instance, enhancing BTLA/HVEM interaction could reduce the proliferation and differentiation of $\mathrm{V} \gamma 9 \mathrm{~V} \delta 2 \mathrm{~T}$ cells that are involved in tumor immune surveillance. ${ }^{24}$ Zhang et $a l^{8}$ reported that overexpression of HVEM in ovarian cancer cells inhibited proliferation and immune functions of T cells, while HVEMsilenced cancer cells had increased the number of activated $\mathrm{T}$ cells as well as the secretion of TNF- $\alpha$ and IFN- $\gamma$, which in turn promoted apoptosis of cancer cells. In general, the above data suggested that BTLA and HVEM may be important factors in the physiopathology of immune regulation and may also have an effect on tumor progression and prognosis. 
Nowadays, molecular characterization of gastric adenocarcinoma has been classified into four distinct subtypes: Epstein-Barr virus (EBV) infected tumors, microsatellite unstable tumors, genomically stable tumors, and chromosomally unstable tumors. ${ }^{25}$ Certain progress has been made in immunology research. PD-L1 expression has been proved to be associated with mismatch repair (MMR) deficiency and EBV positivity in gastric cancer. ${ }^{18}$ Recently, it has been clearly illustrated that the genetic mechanism of immune escape is caused by structural variations of the PD-L1 gene, ${ }^{26}$ which may facilitate the exploration of new generation immune modulators. Hence, further basic studies are required to elucidate the underlying BTLA/HVEM mutation mechanism and evaluate the value of blockade of this pathway in specified subtypes of gastric cancer. Eventually, immunotherapy with anti-BTLA/HVEM monoclonal antibodies might be a viable treatment option for patients with gastric cancer.

\section{Conclusion}

In summary, we demonstrated, for the first time, the correlation of BTLA, HVEM, and gastric cancer. The expression of BTLA and HVEM is increased in most gastric cancers, which is associated with its progression and poor prognosis. BTLA and HVEM might be novel prognostic indicators, and the BTLA/HVEM pathway is considered to be a promising therapeutic target against gastric cancer.

\section{Acknowledgment}

We thank all the patients, as well as their families, who participated in this study. We are also grateful to Qi Huang and Shuli Tang for their technical help and fruitful discussions.

\section{Disclosure}

The authors report no conflicts of interest in this work.

\section{References}

1. Ferlay J, Soerjomataram I, Dikshit R, et al. Cancer incidence and mortality worldwide: sources, methods and major patterns in GLOBOCAN 2012. Int J Cancer. 2015;136(5):E359-E386.

2. Pasero C, Speiser DE, Derre L, Olive D. The HVEM network: new directions in targeting novel costimulatory/co-inhibitory molecules for cancer therapy. Curr Opin Pharmacol. 2012;12(4):478-485.

3. Topalian SL, Hodi FS, Brahmer JR, et al. Safety, activity, and immune correlates of anti-PD-1 antibody in cancer. N Engl J Med. 2012;366(26): 2443-2454.

4. Topalian SL, Drake CG, Pardoll DM. Immune checkpoint blockade: a common denominator approach to cancer therapy. Cancer Cell. 2015; 27(4):450-461.

5. Murphy TL, Murphy KM. Slow down and survive: enigmatic immunoregulation by BTLA and HVEM. Annu Rev Immunol. 2010;28:389-411.

6. Haymaker CL, Wu RC, Ritthipichai K, et al. BTLA marks a lessdifferentiated tumor-infiltrating lymphocyte subset in melanoma with enhanced survival properties. Oncoimmunology. 2015;4(8):e1014246.
7. Inoue T, Sho M, Yasuda S, et al. HVEM expression contributes to tumor progression and prognosis in human colorectal cancer. Anticancer Res. 2015;35(3):1361-1367.

8. Zhang T, Ye L, Han L, He Q, Zhu J. Knockdown of HVEM, a lymphocyte regulator gene, in ovarian cancer cells increases sensitivity to activated T cells. Oncol Res. 2016;24(3):189-196.

9. Migita K, Sho M, Shimada K, et al. Significant involvement of herpesvirus entry mediator in human esophageal squamous cell carcinoma. Cancer. 2014;120(6):808-817.

10. Li D, Fu Z, Chen S, et al. HVEM gene polymorphisms are associated with sporadic breast cancer in Chinese women. PLoS One. 2013;8(8): e71040.

11. del Rio ML, Lucas CL, Buhler L, Rayat G, Rodriguez-Barbosa JI. HVEM/LIGHT/BTLA/CD160 cosignaling pathways as targets for immune regulation. J Leukoc Biol. 2010;87(2):223-235.

12. Sedy JR, Gavrieli M, Potter KG, et al. B and T lymphocyte attenuator regulates $\mathrm{T}$-cell activation through interaction with herpesvirus entry mediator. Nat Immunol. 2005;6(1):90-98.

13. Gonzalez LC, Loyet KM, Calemine-Fenaux J, et al. A coreceptor interaction between the CD28 and TNF receptor family members B and T lymphocyte attenuator and herpesvirus entry mediator. Proc Natl Acad Sci U S A. 2005;102(4):1116-1121.

14. Pasero C, Olive D. Interfering with coinhibitory molecules: BTLA/HVEM as new targets to enhance anti-tumor immunity. Immunol Lett. 2013; 151(1-2):71-75.

15. Shui JW, Kronenberg M. HVEM: an unusual TNF receptor family member important for mucosal innate immune responses to microbes. Gut Microbes. 2013;4(2):146-151.

16. Edge SB, Compton CC. The American Joint Committee on Cancer: the 7 th edition of the AJCC cancer staging manual and the future of TNM. Ann Surg Oncol. 2010;17(6):1471-1474.

17. Feng XY, Wen XZ, Tan XJ, et al. Ectopic expression of B and T lymphocyte attenuator in gastric cancer: a potential independent prognostic factor in patients with gastric cancer. Mol Med Rep. 2015;11(1): 658-664.

18. Kawazoe A, Kuwata T, Kuboki Y, et al. Clinicopathological features of programmed death ligand 1 expression with tumor-infiltrating lymphocyte, mismatch repair, and Epstein-Barr virus status in a large cohort of gastric cancer patients. Gastric Cancer. Epub 2016 Sep 14.

19. Lesokhin AM, Ansell SM, Armand P, et al. Nivolumab in patients with relapsed or refractory hematologic malignancy: preliminary results of a Phase Ib study. J Clin Oncol. 2016;34(23):2698-2704.

20. Abdel-Rahman O. Immune checkpoints aberrations and gastric cancer; assessment of prognostic value and evaluation of therapeutic potentials. Crit Rev Oncol Hematol. 2016;97:65-71.

21. Heo SK, Ju SA, Kim GY, et al. The presence of high level soluble herpes virus entry mediator in sera of gastric cancer patients. Exp Mol Med. 2012;44(2):149-158.

22. M'Hidi H, Thibult ML, Chetaille B, et al. High expression of the inhibitory receptor BTLA in T-follicular helper cells and in B-cell small lymphocytic lymphoma/chronic lymphocytic leukemia. Am J Clin Pathol. 2009;132(4):589-596.

23. Launay E, Pangault C, Bertrand P, et al. High rate of TNFRSF14 gene alterations related to $1 \mathrm{p} 36$ region in de novo follicular lymphoma and impact on prognosis. Leukemia. 2012;26(3):559-562.

24. Gertner-Dardenne J, Fauriat C, Orlanducci F, et al. The co-receptor BTLA negatively regulates human Vgamma9Vdelta2 T-cell proliferation: a potential way of immune escape for lymphoma cells. Blood. 2013;122(6):922-931.

25. Cancer Genome Atlas Research Network. Comprehensive molecular characterization of gastric adenocarcinoma. Nature. 2014; 513(7517):202-209.

26. Kataoka K, Shiraishi Y, Takeda Y, et al. Aberrant PD-L1 expression through 3'-UTR disruption in multiple cancers. Nature. 2016; 534(7607):402-406. 


\section{Publish your work in this journal}

OncoTargets and Therapy is an international, peer-reviewed, open access journal focusing on the pathological basis of all cancers, potential targets for therapy and treatment protocols employed to improve the management of cancer patients. The journal also focuses on the impact of management programs and new therapeutic agents and protocols on

patient perspectives such as quality of life, adherence and satisfaction. The manuscript management system is completely online and includes a very quick and fair peer-review system, which is all easy to use. Visit http://www.dovepress.com/testimonials.php to read real quotes from published authors.

Submit your manuscript here: http://www.dovepress.com/oncotargets-and-therapy-journal 